\section{¿RETORNO AL PASADO? COMERCIO O DIFUSIÓN EN LOS ANÁLISIS DE LOS SISTEMAS MUNDIALES ANTIGUOS}

\author{
BACK TO THE PAST?. TRADE OR \\ DIFFUSION IN ANCIENT WORLD \\ SYSTEMS ANALYSIS
}

\section{ALFREDO MEDEROS MARTÍN (*)}

\section{RESUMEN}

Desde fines de los años ochenta un número creciente de aproximaciones al comercio en el Mediterráneo durante la Prehistoria Reciente está enfatizando la presencia de Sistemas Mundiales Antiguos lo cual podría interpretarse como una vuelta al difusionismo. Se señala el contexto sociopolítico europeo y los nuevos estudios de procedencia de los productos como la razón de su popularidad, sus bases teóricas en el materialismo de enfoque circulacionista junto a los peligros y beneficios de dichos enfoques.

\begin{abstract}
Since the end of the eighties, an increasing number of approaches to trade in the Mediterranean during the late Prehistory have emphasized the presence of Ancient World Systems, a frend which could be explained as a return to diffusionism. We show the European sociopolitical context and new provenance studies as the reason for its new popularity, its theoretical role in circulationist materialism, together with the danger and benefit in this kind of approach.
\end{abstract}

(*) Becario Postdoctoral de la Dirección General de Investigación Científica y Técnica. Ministerio de Educación y Ciencia en el Department of Archaeology, University of Bristol, 11 Woodland Road, BS8 1TB Bristol, U.K., Tlf \& Fax 44 1179288678 .

El artículo fue remitido en su versión final el 30-X-95.
Palabras clave: Sistemas Mundiales. Difusión. Comercio. Europa.

Key words: World Systems. Diffusion. Trade. Europe.

\section{INTRODUCCIÓN}

Con el inicio de la década de los años noventa, una nueva estrategia de análisis del comercio está ganando protagonismo dentro del estudio de la Prehistoria del Mediterráneo y Próximo Oriente, interpretando la presencia de intercambios dentro de un contexto supranacional íntimamente relacionado, denominado «World System» o Sistema Mundial. El elemento determinante en este tipo de análisis es que todos los elementos integrados en el mismo interactúan entre sí, y que cada fenómeno, aunque sea indirectamente, resulta explicable dentro de la totalidad del Sistema Mundial. El individuo o los colectivos, consecuentemente, se ven relativamente mediatizados por el Sistema Mundial en el que se insertan, y sus actos tienen una última explicación dentro del mismo.

Las relaciones de dependencia entre centro y periferia del Sistema Mundial es el rasgo más característico del mismo, y son explicables por la presencia de un intercambio desigual, a priori, entre un centro más avanzado en la producción de productos manufacturados, parte de los cuales son intercambiados con su periferia a cambio 
de materias primas exóticas o productos semielaborados. Este intercambio desigual favorece los procesos de acumulación de las élites del centro, pero no debemos olvidar que esta acumulación de riqueza procedería aún en mayor medida de la obtención de tributos, impuestos, botín, etc., en territorios donde junto a una dependencia económica coexistió un control militar o político de los mismos. o simplemente en áreas que eran objeto de razzias ocasionales.

El objetivo de este trabajo es analizar algunas de las bases teóricas de esta nueva estrategia de análisis en sus aplicaciones a la Prehistoria Reciente del Mediterráneo, en el marco del contexto sociopolítico contemporáneo en el que se está desarrollando.

\section{¿EL RETORNO DE LOS DIFUSIONISTAS?}

Este fenómeno, que podría interpretarse por algunos investigadores como una nueva versión del difusionismo, ya que centra su mayor interés en la presencia de algún tipo de contacto comercial entre dos territorios relativamente distantes, aún cuando el número de evidencias cuantificables sea relativamente escaso, tiene su fundamento en la relativa crisis de la New Archaeology, frente al empuje de las estrategias post-procesuales, y en el progresivo desarrollo de nuevas técnicas analíticas para interpretar la procedencia de los productos. En este sentido, hay que agradecer el atrevimiento de $\mathrm{A}$. Sherratt (1993a: 249), quien tras preguntarse si los medios de esta estrategia suenan muy próximos al difusionismo responde, «sí, pero, ¿por qué no?».

Sin embargo, existen notables diferencias entre ambas estrategias de investigación. El difusionismo parte del principio de que el ser humano es un sujeto pasivo poco partidario o contrario al cambio, escasamente inventivo o imaginativo, y capaz de rechazar o incluso eliminar a los innovadores que puedan poner en cuestión aspectos básicos de su modo y medios de vida. Este principio resultaba asumible desde el momento en que se presuponía la presencia de centros culturales más desarrollados que serían los que estimulaban el cambio cultural.

El desequilibrio en la variedad y riqueza del registro artefactual, arquitectónico y literario del Mediterráneo Oriental y Próximo Oriente respecto al Mediterráneo Occidental o el Centro y Norte de Europa aportaba el argumento bási- co para sustentar estas premisas. La posibilidad de reconstruir las secuencias de la Europa Occidental y Septentrional a partir de importaciones o imitaciones de artefactos típicos supuestamente bien fechados en el Mediterráneo Oriental a partir de la cronología histórica, implicaba poder disponer de la cronología comparada absoluta, entonces única alternativa para reconstruir las secuencias históricas occidentales, ante la inexistencia del método de datación por carbono 14.

Interpretar los Sistemas Mundiales como una nueva versión refinada del neodifusionismo no resulta una apreciación correcta. Ahora no se trata de analizar la presencia de algún tipo de intercambios a larga distancia, inclusive con una red de contactos a través de la cual circulan regularmente cierto número de productos, sino de determinar si existen relaciones de dependencia económica entre dos regiones vecinas o relativamente distantes por la presencia de relaciones de intercambio desiguales.

La cuestión crucial a determinar en este tipo de análisis es a partir de cuando puede esgrimirse la presencia de estas relaciones de dependencia en función del registro arqueológico o literario disponible. No basta con utilizar evidencias puntuales sobre algún tipo de intercambio comercial, y tampoco con la mera acumulación de artefactos dispares que son enumerados en algunos artículos, sin tratar de aportar una cuantificación de los mismos. Finalmente, la intuición de considerar que los artefactos ya documentados son una punta de «iceberg» dentro del registro arqueológico es admisible desde el punto de vista de una hipótesis de trabajo, pero no como una evidencia empírica demostrada.

Consecuentemente, los límites del registro arqueológico abren siempre la duda ante este tipo de argumentaciones, y a medida que se incrementa la distancia entre dos regiones y se debilitan las posibles evidencias de intercambios comerciales, se acentua la fragilidad de las pruebas que pueden esgrimir los autores que las defienden.

Es entonces cuando surge el dilema de que si priorizamos el siempre modesto volúmen de intercambios, y el fenómeno de aculturación que pudo estar interactuando paralelamente, podemos estar infravalorando registros artefactuales mayoritariamente autóctonos insertos en procesos autónomos de desarrollo de las diferentes Formaciones Sociales objeto de análisis, pudien- 
do surgir entonces veladas acusaciones de neodifusionismo de nuevo cuño.

En cierto modo, todos estos planteamientos rompen con el paradigma autoctonista actualmente vigente en la investigación de la Prehistoria de la Europa Occidental. Es a mediados de los sesenta, con la lectura de la tesis doctoral de C. Renfrew, "The Neolithic and Early Bronze Age cultures of the Cyclades and their external relations» (1965), reelaborada para su publicación (Renfrew, 1972), cuando se abría la brecha más profunda contra los enfoques difusionistas hasta entonces dominantes, al plantear un desarrollo autónomo del Egeo, Cicládicos, Minoicos y Micénicos, frente a los centros urbanos estatales del Próximo Oriente, de acuerdo con los enfoques autoctonistas propios de las estrategias procesuales, liberándose la primera civilización europea de su deuda con áreas asiáticas y africanas, que acababan de buscar en la independencia un desarrollo político autónomo de las antiguas metrópolis colonialistas europeas.

Su estrategia de ataque contra los argumentos tradicionales se orientó hacia cuatro objetivos:

1) La interacción tradicionalmente esgrimida entre la denominada "Cultura» Wessex I, y su monumento más representativo, Stonehenge, con la Grecia Micénica del Bronce Final, que implicaba un fuerte impacto social en el gran público británico (Renfrew, 1968).

2) La supuesta arribada de colonizadores egeos o del Próximo Oriente al Mediterráneo Occidental, y concretamente la Península Ibérica (Renfrew, 1967) y la Península Itálica (Renfrew y Whitehouse, 1974), cuya fragilidad argumental puso en evidencia a partir de su conocimiento directo de una de las tradicionales presuntas áreas de partida, las islas Cícladas.

3) La necesidad de buscar técnicas analíticas adecuadas que confirmasen, o refutasen, el tradicional recurso a paralelos tipológicos o estilísticos, caso de la obsidiana (Cann y Renfrew, 1964), el mármol (Renfrew y Peacey, 1968) o las cuentas de fayenza (Newton y Renfrew, 1970).

4) Y la utilización de las dataciones radiocarbónicas calibradas por dendrocronología, que demostraba la primacía de muchos fenómenos de la Europa Occidental con respecto a sus paralelos en el Egeo o el Próximo Oriente, particularmente del megalitismo (Renfrew, 1970).

Del triunfo de sus argumentos a nivel continental europeo no cabe la menor duda, a pesar de la osadía de su empresa. inclusive tardíamente en el Estado español, donde hemos vivido una corriente profundamente antidifusionista desde mediados de los años ochenta.

Por esas paradojas del destino, el desarrollo de nuevas técnicas analíticas, algunas de las cuales inicialmente dieron lugar a uno de los mayores fracasos científicos dentro de las estrategias difusionistas, el comercio del metal defendido por el grupo de Stuttgart que publicó los primeros volúmenes de los «Studien zu den Anfängen der Metallurgie» (Junghans et alii, 1960) bajo la impronta de E. Sangmeister, ha ido proporcionando progresivamente un mayor corpus de datos sobre el intercambio de distintos productos entre el Mediterráneo Central y la India. Los análisis de isótopos de plomo para dilucidar el comercio del cobre, bronce, plata, o plomo, los de absorción de rayos $\mathrm{X}$ y espectro de infrarrojos para el ámbar, los análisis de isótopos estables para el mármol, la petrografía de láminas delgadas y la espectrometría de fluorescencia de rayos $\mathrm{X}$ para las rocas, o esta última para la fayenza, los de activación neutrónica y petrográficos en los análisis de cerámica, etc., en suma los denominados estudios de procedencia, van aportando un corpus de datos que, una vez que se tengan suficientes muestras de un gran número de regiones, abrirá aún nuevas perspectivas de investigación, aunque todas estas analíticas progresivamente van mostrando los límites en su precisión.

En este contexto, la aparición de cerámica micénica del Heládico Final III A2 o IIIB en la Península Ibérica, concretamente en el Llanete de los Moros (Montoro, Córdoba) (Martín de la Cruz, 1987: 63), marcó un hito al abrir la posibilidad de que existiera algún tipo de comercio entre el Egeo y el Mediterráneo Occidental al menos durante el Bronce Final, siendo ratificado a nivel institucional por la Exposición «El Mundo Micénico» en Madrid (1992), donde se presentó a los micénicos como la primera civilización europea que interrelacionó el Mediterráneo Oriental con el Mediterráneo Central y Occidental.

\section{EL NUEVO CONTEXTO POLÍTICO EUROPEO}

Esta rápida reacción de las instituciones públicas ante un descubrimiento arqueológico 
pone en evidencia. en este caso por los gobiernos de España y Grecia, que vivimos en un nuevo contexto sociopolítico al que difícilmente resulta ajena la arqueología europea.

A partir de la $3^{\text {a }}$ Conferencia Europea de Ministros reponsables del Patrimonio Cultural (La Valletta, Malta, enero 1992), se presentó el $1^{\circ}$ Plan Europeo de Arqueología en el cual se tomó como lema «La Edad del Bronce: la primera edad de oro de Europa", que en su acepción anglosajona incluye nuestro Calcolítico, Bronce y colonizaciones, circa cal. 300()500 AC, a desarrollarse entre el otoño de 1994 y el verano de 1996 . Tal como refleja el catálogo de dicho programa, «La Edad del Bronce fue un apasionante periodo de la historia de Europa cuando los contactos entre las diferentes partes de la gran Europa comenzaron a desarrollarse, reflejando la moderna interacción entre los estados miembros del Consejo de Europa». "Ella representa la primera industrialización en la historia de Europa...» con «...una dependencia del comercio internacional de materias primas importadas. Esta expansión del comercio internacional (...) aceleró la marcha del cambio en Europa...». "Fue durante la Edad del Bronce cuando la mayoría de Europa entró en la arena de la historia mundial. (...) en una nueva era de comunicaciones internacionales e interdependencia la cual ha permanecido como una condición básica desde entonces».

Como puede observarse, el nuevo contexto europeo exige comenzar a ir sentado las bases para reinterpretar la historia de Europa desde un marco de interacción entre los distintos Estados miembros que la componen. Dentro de esta nueva realidad, los arqueólogos habrán de prestar especial interés en aportar a la Prehistoria europea nuevas evidencias de relaciones supranacionales que ayuden a fortalecer los nuevos lazos económicos y políticos.

Este tipo de enfoques, que en ocasiones tienden a ser minusvalorados por los profesionales con una educación superior universitaria, choca con la evidencia práctica reflejada recientemente, por ejemplo, en una encuesta a los estudiantes entre 11 y 14 años de las escuelas públicas de Gran Bretaña, según la cual a 50 años del final de la II Guerra Mundial, un $25 \%$ no identificó a A. Hitler, un $36 \%$ desconocía quien fue W. Churchill, un $40 \%$ el día de la victoria de los aliados en Europa, un 60 \% el Holocausto de los judíos o de que bando había luchado Italia, y un $90 \%$ al general Montgomery, a pesar de tratarse de un país que sufrió duramente pero ganó la guerra, participaron en ella buena parte de sus abuelos y hay una numerosa serie de filmes bélicos sobre el tema que son repuestos por las televisiones cada cierto tiempo.

En este nuevo marco histórico habría que interpretar las propuestas de crear un Instituto Europeo de Arqueología, los cada vez más claros intentos de reconvertir la reunión anual del Theoretical Archaeological Group, en un EuroTAG, tal como se presentó la reunión en Southampton en diciembre de 1992 (Ucko, 1995), o la creación de la European Association of Archaeologist y del Journal of European Archaeology, también en diciembre de 1992 todos ellos, como puede apreciarse, un mes antes del comienzo de Mercado Unico Europeo en enero de 1993.

Es preciso matizar que lo que se trata de reflejar no es una estrecha relación de convergencia de intereses, sino la progresiva adaptación de una ciencia social como la Arqueología a una nueva realidad política, en la búsqueda de recursos financieros para sustentar líneas de investigación, y ante la necesidad de crear nuevas organizaciones que ayuden a defender mejor los intereses comunes de la profesión a nivel europeo.

En todo caso, sí se aprecia que en el futuro los soportes analíticos que sustentan estas estrategias de investigación, probablemente gozarán de cauces de financiación adecuados $o$, al menos, con dotaciones mayores o tan relevantes como otras líneas de trabajo. En este sentido, es interesante plantear que los estudios sobre procedencia de metales a partir de isótopos de plomo, desarrollados en el Mediterráneo Oriental por investigadores de la Universidad de Oxford (Gale y Stos-Gale, 1982), han gozado de una especial financiación en los últimos años por parte del «Science and Engineering Research Council», que si bien resulta lógico por su carácter innovador, priorizado por dicha comisión, y los aparentemente espectaculares resultados obtenidos, no conviene olvidar que ha sido el segundo programa de investigación arqueológica mejor financiado en los últimos años en el Reino Unido, detrás de la Unidad de Espectroscopía de Masas de Aceleración (A.M.S.) del «Research Laboratory for Archaeology» en Oxford. 


\section{LOS ORIGENES DE LOS SISTEMAS MUNDIALES ANTIGUOS Y SU PRECURSOR: VERE GORDON CHILDE}

El antecedente más obvio de la presencia de Sistemas Mundiales desde el Calcolítico en Egipto, Mesopotamia y la India es la obra de V.G. Childe. Un repaso a sus trabajos más representativos resulta clarificador, y pone en evidencia que buena parte de las nuevas propuestas siguen sus pasos, ahora con un soporte analítico cada vez más adecuado, aunque cuantitativamente con datos aún insuficientes.

$\mathrm{Su}$ criterio determinante es que «la completa autosuficiencia económica tal vez no se ha logrado en ninguna parte», pero además sucedía que «a pesar de su abundancia de alimentos, los valles de aluvión son extraordinariamente pobres en otras materias primas fundamentales", caso de la piedra, la madera, el sílex, las rocas pulimentadas, el cobre, etc. Partiendo de esta necesaria premisa, «los habitantes de Egipto, Sumer y la cuenca del Indo se vieron obligados a organizar algún sistema regular de comercio o de trueque, para asegurarse el abastecimiento de materias primas esenciales. La fertilidad de las tierras dió a sus habitantes los medios de satisfacer su necesidad de importaciones». Este «intercambio se hizo más estrecho que nunca en el momento de ocurrir la revolución (urbana), o inmediatamente después de ella». Pronto «El excedente (...) también debió servir para sostener un cuerpo de comerciantes (...) soldados para proteger (...) los convoyes (...) los escribas para llevar registro de las transacciones cada vez más numerosas y complicadas y los funcionarios del Estado para conciliar los intereses en conflicto" (Childe, 1954: 118, 189190, 224).

«El tráfico regular establecido sobre estas bases entre los mercados urbanos y las minas, o canteras, o bosques, proporcionó el mecanismo para la distribución de materias primas a las regiones intermedias, demasiado pobres para mantener por sí solas un comercio regular».

«Al mismo tiempo, el tráfico regular con (los) centros urbanos debió promover una aproximación a la organización civilizada en las regiones productoras de materias primas, y en las intermedias por donde pasaba la vía comercial. El representante de la comunidad local (...) debió encontrarse controlando un superavit de riquezas que ya no dependía (...) de los dones habituales de los miembros de la comunidad. De esta forma obtuvo una cierta independencia económica".

De tal forma que «hacia el 2500 a.C. tenemos pruebas concretas de una red comercial que enlazaba todo el área desde el Tigris al Indo y al Oxus, y que se extendía al Eufrates y hasta el Nilo» (Childe, 1986: 203, 291), creándose «una relación continua, que comprendía el intercambio de mercancías, ideas y artesanos" (Childe, 1954: 225).

Consecuentemente, «la revolución urbana (...) en los valles del Nilo, del Tigris y del Eufrates, y del Indo" acabó "creando en el terreno económico la primera acumulación de capital necesario». De tal forma que el «establecimiento en el Egeo de una economía de la Edad del Bronce se produjo, de hecho, gracias al capital oriental (...) debido a la acumulación de las reservas esenciales y al descubrimiento y perfeccionamiento de nuevas técnicas y métodos en respuesta a una demanda de los mercados orientales».

Pronto «las comunidades del primitivo Egeo eran ya lo bastante ricas como para ser centros secundarios de demanda, así como mercados para los productos de la Europa bárbara».

"Andando el tiempo, también Europa contribuiría a satisfacer la demanda oriental a base de materias primas y de artículos de lujo, de forma que las sociedades neolíticas europeas pudieron beneficiarse de una industria cuyo establecimiento había sido posible gracias a los mercados egipcio y mesopotámico" (Childe, 1978: 94, 123, 128).

Parte de estas premisas de Childe acabarán por ser retomadas por investigadores como C.C. Lamberg-Karlovsky y M. Tosi (1973) o P. Kohl (1978), que resaltarán el papel intermediario de la llanura iraní y del Asia Central, respecto a Mesopotamia y el valle del Indo.

En todo caso, la base determinante de este tipo de aproximaciones, y de ahí la actualidad de las propuestas de Childe, es su intento de aportar análisis globales de los procesos históricos objeto de estudio, rompiendo la compartimentación que los distintos conjuntos artefactuales regionales y nacionales van creando con el incremento de la información disponible, y que consolida la multiplicidad lingüística, de tal forma que en la actualidad atenaza seriamente a la investigación arqueológica al contreñirse la gran mayoría de los estudios, incluso de síntesis, 
como mucho a un análisis regional de una fase temporal concreta.

\section{LOS ENFOQUES CIRCULACIONISTAS}

La importancia de los procesos de circulación en la economía capitalista es desarrollada por K. Marx (1959) en los libros II y III de El Capital; se refleja en el debate presente en la correspondencia entre F. Engels y K. Kautsky en la primera mitad de los años 80 del siglo XIX, y tiene su continuidad en la obra de Lenin (1960), quien al igual que Marx hace particular énfasis en la importancia del capital financiero como instrumento de sometimiento de la independencia política de los países al entonces dominante capitalismo británico.

Un nuevo desarrollo sobre la expansión del capitalismo es ofrecido por R. Luxemburg (1967) en su polémica con $\mathrm{O}$. Bauer. Luxemburg se inclina por buscar en la necesidad de nuevos mercados el fenómeno estimulador del capitalismo imperialista, mientras que para Bauer la razón habría que encontrarla en la necesidad de reclutar nueva fuerza de trabajo de la cual extraer plusvalías, argumento rechazado por Luxemburg ya que entonces se seguía produciendo un permanente éxodo migratorio de mano de obra proletaria sobrante desde los países capitalistas hacia las colonias o nuevos estados independientes americanos.

El estudio de esta problemática recibió en los años sesenta la denominación de Teoría de la Dependencia al incentivarse estos análisis, tras la descolonización progresiva después de la II Guerra Mundial, orientados a estudiar los procesos de desarrollo en la periferia, actual Tercer Mundo, del sistema capitalista.

Tras el precursor trabajo de P. Baran (1959), se desencadenará una polémica (Sweezy et alii, 1977) entre M. Dobb y P. Sweezy acerca del elemento determinante para la transición al capitalismo, al defender este último que fue la ampliación de los mercados hacia Africa, Asia y América el elemento determinante en la transformación de las relaciones de producción, frente a la tesis clásica con los enfoques materialistas de Dobb, partidario de una expansión mundial $a$ posteriori de la transformación de dichas relaciones en Europa; retomando parte de la vieja discusión.
El papel desempeñado por la periferia, y su subdesarrollo generado por la presencia de un intercambio desigual de materias primas a cambio de productos manufacturados como elemento fundamental en el desarrollo de la acumulación del capital en Europa Occidental y posteriormente en los Estados Unidos, ha sido la tesis defendida por A.G. Frank $(1973,1979)$ y S. Amin (1974), destacando la labor realizada por A. Emmanuel (1973) para individualizar las características que definen el intercambio desigual.

En esta línea, la obra que ha gozado de una mayor repercusión ha sido la tesis defendida por I. Wallerstein 1979), particularmente en su primer volumen, partidario de la existencia de un Sistema Mundial desde el siglo XVI d.C. En su trabajo se aprecia la huella de uno de los miembros de la escuela de los «Annales», F. Braudel (1994), particularmente la incorporación de sus nociones de la economía-mundo mediterránea y de los ciclos de larga y corta duración. Un dato significativo es que Wallerstein (1988: 30) pone su énfasis en que el Sistema Mundial capitalista no se ha expansionado en búsqueda de nuevos mercados, ya que las áreas externas al sistema en general carecían básicamente de capacidad adquisitiva para comprar productos manufacturados, y en parte, porque no les hacían falta, planteando que el fin último de esta expansión ha sido la búsqueda de mano de obra a bajo coste que redujera los costes de producción y contribuyese, además, a no incrementar los salarios de los trabajadores europeos.

Wallerstein mantiene en su planteamiento los enfoques del sustantivismo primitivista o marginalista de la antropología económica, que considera inaplicables al pasado las categorías elaboradas para economías de sistemas capitalistas con mercado. Para ello se adhiere a las tesis neoricardianas propugnadas por K. Polanyi (Polanyi et alii, 1976: 289-316), seguidas por G. Dalton (1976), que distinguen economías organizadas bajo la reciprocidad y el parentesco, otras articuladas bajo la redistribución por la élite gobernante y, finalmente, aquéllas en las que el mercado funciona como el elemento determinante de la economía. Esta tesis, por lo tanto, rechaza los planteamientos formalistas que consideran aplicables a las sociedades pretéritas categorías propias de la economía capitalista como la competición por el beneficio, el valor, el precio o la acumulación propugnadas entre 
otros por M.J. Herskovitz (1954) o E. y H. Schneider (1957).

Sin embargo, este "rígido" marco temporal ceñido al capitalismo moderno fue pronto matizado, cuando J. Schneider (1977: 25) se planteó si «¿Había un Sistema Mundial Pre-capitalista?». En dicho trabajo defendió su presencia desde el Calcolítico mesopotámico a partir del intercambio desigual de un centro exportador de manufacturas, particularmente tejidos, y acumuladora de metales preciosos recibidos como pago de las áreas periféricas, a veces junto con esclavos. No obstante, Wallerstein (1991: 192) continúa rechazando retrotraer su Sistema Mundial, admitiendo como mucho el límite del 1450 dC.

El Symposio celebrado en Copenhagen (1977) sobre «Empires in the Ancient World" (Larsen, 1979) ejemplificó en un artículo del asiriologo M.T. Larsen las posibilidades inherentes al modelo de Wallerstein para el ámbito mesopotámico, mientras K. Ekholm y J. Friedman (1979) propusieron ya entonces la presencia de unos Sistemas Mundiales Antiguos. Esta línea de trabajo continuó en la conferencia de Aarhus, Dinamarca (1980) sobre «Relations between the Near East, the Mediterranean World and Europe. 3rd to 1st millenium B.C.», sin embargo no será publicada hasta siete años después (Rowlands et alii, 1987).

Pese a la creciente bibliografía, a nivel práctico las propuestas concretas no abundan, destacando para el Nuevo Mundo las de R. Pailes y J. Whitecotton (1979) y R. Banton y G. Feinman (1984) que comienzan a proponer la presencia de un Sistema Mundial en Mesoamérica, aún por desarrollar.

En el caso del Viejo Mundo, P. Kohl (1989) hace lo propio para Mesopotamia, Asia Central y la Meseta Iraní, y C. Edens (1992) lo interconecta con el Golfo de Arabia y Golfo de Omán, apuntando sus relaciones, aún por exponer en detalle, con el Valle del Indo, siendo el primer investigador que aporta datos cuantitativos para determinar el volumen de intercambios, y las fluctuaciones que estos presentan.

Respecto al continente europeo han sido A. y S. Sherratt (1991) quienes lo plantean inicialmente para el Mediterráneo Oriental entre el 2500-1000 AC, en la conferencia sobre «Bronze Age Trade in the Mediterranean" (Oxford, 1989), y después a la totalidad del Mediterráneo entre el 1000-500 AC (S. y A. Sherratt, 1993), o al resto de Europa como área marginal entre 2500)-500 AC (Sherratt, 1993). En este sentido, las aproximaciones a los Sistemas Mundiales a partir de la Edad del Hierro, ya se habían producido previamente desde el trabajo de S. Frankestein y M. Rowlands (1978) para el sur de Alemania, e in extenso en monografías de investigadores especialistas en tal fase temporal, caso de P. Brun (1987), 850-450 aC, o B. Cunliffe (1988), 530 BC-10 BC/43 AD.

Un enfoque particular está presente en $\mathrm{K}$. Randsborg (1991, 1992), quien dado el papel relativamente marginal de Dinamarca respecto al proceso histórico que se desarrolla en el Mediterráneo, propone una línea de continuidad entre las poblaciones del Norte de Europa desde la Edad del Bronce, Hierro (c. 400 AC) hasta el Imperio Carolingio, como cierto sustrato de la actual Europa comunitaria.

Un dato interesante de los estudios sobre los Sistemas Mundiales es que se trata de un enfoque definido inicialmente por sociólogos, con formación histórica y antropológica, Frank, Wallerstein y Friedman, que ha sido retomado a nivel práctico por investigadores próximos a algunas de las mayores bibliotecas arqueológicas actualmente disponibles, Harvard (Lamberg-Karlovsky, Kohl, Edens) y Oxford (Cunliffe, los Sherratt), quienes disponen de suficientes recursos bibliográficos para remitirse a los trabajos originales de áreas geográficas muy dispersas, desde la Península Ibérica hasta la India, lo que obviamente no está al alcance de muchos investigadores.

Sin embargo, se trata de estrategias diferenciadas de análisis. El interés de sociólogos como Frank o Gills es prioritariamente obtener en el pasado respuestas que expliquen las características que definen al actual sistema capitalista. Por tal circunstancia, es importante definir la presencia incluso en el pasado de un único Sistema Mundial Antiguo en el cual se suceden ciclos económicos de crecimiento seguidos de otros de estancamiento, que tienen su reflejo igualmente en la construcción y posterior decadencia de centros políticos hegemónicos. Además, en esta ambiciosa propuesta, se trata de proponer una lectura renovada del materialismo histórico (Gills y Frank, 1990: 31) en la cual, frente a la tradicional transición de sucesivos Modos de Producción, se propone desarrollar ahora un estudio de transiciones entre cuatro Modos de Acumulación de excedentes, estatal, privada, 
dominante estatal, y dominante privada, en la cual juega un papel prioritario la progresión desde la acumulación primitiva de excedentes iniciada en la Prehistoria Reciente hasta la actual acumulación masiva de capital en pocas personas de un número reducido de países del actual centro.

Por el contrario, prehistoriadores como Kohl (1987: 16, 23) han defendido la presencia de una serie de Sistemas Mundiales que interactuaban entre sí, pero con un elevado grado de independencia, lo que creemos se ajusta mejor al actual registro arqueológico disponible. De esta manera, se interpretaría más coherentemente la articulación entre subsistemas que parecen tener un notable grado de autonomía, como el Egeo, Egipto, Levante, Mesopotamia, Golfo ArábigoGolfo de Omán, Turkestán Occidental o Valle del Indo entre otros, hipótesis de trabajo a la que parece que también va inclinándose progresivamente Frank (1993: 387, 425) cuando ahora habla de un Sistema Mundial Central dentro de los Sistemas Mundiales Antiguos.

En segundo lugar, se trata de una alternativa interesante para orientalistas familiarizados con los textos escritos del Próximo Oriente (Larsen, 1979; Liverani, 1987), ya que dichas fuentes aportan un volumen de información sobre intercambios y presencia de relaciones diplomáticas entre países que nunca podremos encontrar en una magnitud similar a nivel arqueológico, siendo particularmente importantes en lo referente a intercambios de productos orgánicos, cereales, tejidos..., muy difícilmente contrastables por el arqueólogo.

En tercer lugar, ha sido utilizado por Randsborg, quizás sin una amplia formación académica clásica o en la Prehistoria mediterránea, como una alternativa para insertar algunas prehistorias nacionales que resultan periféricas a las pautas marcadas por el Mediterráneo europeo, tratando de salvar los desfases cronológicos que suponen la tardía aparición de las distintas etapas de la metalurgia del cobre, bronce y hierro, y de liberarse de la tradicional dependencia de la secuencia de $\mathrm{O}$. Montelius por las aún escasas dataciones radiocarbónicas disponibles, a veces desde un enfoque actualista discutible que extiende el desarrollo de su Prehistoria, Bronce I-V y Hierro, hasta la disolución del Imperio Romano, con tentaciones de que culminaría en la creación del Imperio de Carlomagno, a modo de sustrato de la actual Europa comunitaria.
Sin embargo, este enfoque actualista de buscar explicaciones contemporáneas en la Prehistoria europea, tiene el inconveniente de que el Imperio Carolingio apenas sobrepasó hacia el norte el límite de Hamburgo, con lo que en la práctica no afectó a Dinamarca ni al resto de los países escandinavos, y realmente lo que hace inconscientemente es abrir caminos a algunos defensores del pangermanismo, "atentando" paralelamente contra algunos principios aparentemente esenciales de la identidad europea, Grecia como cuna de la Democracia o el Imperio Romano como máxima extensión de los principios clásicos occidentales, y hablando más en serio, simplifica en ocasiones la interrelación entre la Edad del Bronce y del Hierro italiana y griega con buena parte de las de centroeuropa, y obviamente, de las propias de Escandinavia (p.ej., Harding, 1984; Bouzek, 1985).

\section{LOS RIESGOS DE LOS ENFOQUES CIRCULACIONISTAS}

Entre los problemas que subyacen en esta estrategia de investigación, podríamos reseñar varios de los más relevantes:

En principio, al enfatizar los procesos de acumulación generados por el comercio de bienes de difícil acceso entre las élites, se minusvalora que la fuente básica de obtención de excedentes sigue proveniendo del campesinado a través de tributos o impuestos, la cual permite su manutención y les posibilita disponer de tales productos para ser intercambiados.

Por otra parte, aunque las élites suelen ser las que detentan finalmente su propiedad o disfrute, no son quienes elaboran los productos manufacturados u obtienen las materias primas que se intercambian, o quienes transportan y comercian con dichos productos en contextos foráneos.

En segundo lugar, se tiende a conceder a las élites el papel de "gestor» en la toma de decisiones que posibilitan tal tipo de intercambios. Como interesadas en obtener determinados productos para detentar públicamente su estatus social, planificarían la elaboración de determinados productos manufacturados por artesanos a tiempo parcial o completo, establecerían relaciones diplomáticas con sus iguales de otras unidades políticas, organizarían expediciones comerciales o militares orientadas a la obtención 
de dichos productos, y una vez conseguidos, se encargarían de distribuirlos mediante pagos o regalos.

Este punto de vista resulta muy grato a los enfoques funcionalistas ya que pone su énfasis en la competición por el estatus social entre las élites, y concretamente entre individuos particulares, como el elemento básico que conduce el proceso histórico, ya que a través de la toma de decisiones personales, el deseo de obtener mayores riquezas o prestigio personal, se crean redes de intercambio, circulan productos, artesanos, esclavos especialistas o información, se desencadenan guerras, o se firman tratados diplomáticos. Consecuentemente, cuando ese énfasis en la competición por el prestigio cesa, o se produce por causas exogénas la ruptura de los canales comerciales creados, léase invasiones p.ej., dichas élites se debilitan y tiende a producirse el colapso de las unidades políticas que dirigen.

En todo caso, tal como plantean Ekholm y Friedman (1979: 48), uno de los elementos esenciales de los Sistemas Mundiales es que, al tratarse de una producción dirigida y restringida a su consumo por las élites dirigentes, las únicas posibilidades de expansión del mercado son el comercio a larga distancia que incorpora nuevos efectivos de las élites locales.

Sin embargo, conviene hacer la matización que probablemente algunos productos tuvieron un consumo más ámplio, caso del cobre o los productos textiles, p.ej., tal como hacen presuponer los volúmenes cuantificables documentados en determinadas fuentes escritas, y ambos tipos de comercio interactúan dentro de los Sistemas Mundiales.

\section{A MODO DE CONCLUSIÓN}

Al llegar a este punto, hemos de preguntarnos finalmente, ¿vale la pena desarrollar este tipo de enfoques circulacionistas?. Desde nuestro punto de vista creemos que sí, pese a los problemas que subyacen en esta estrategia de investigación, tal como hemos planteado.

Dado que partimos de la desventaja de que en periodos como la Prehistoria Reciente de la Península Ibérica nos encontramos ante poblaciones que desconocían la escritura, creemos que resulta una falacia hacer depender de por vida las estrategias de investigación orientadas a estudiar sociedades desiguales exclusivamente de argumentar la posible presencia de unos presuntos impuestos o tributos, modos de propiedad o tenencia de la tierra determinados, etc, que nunca podrán demostrarse adecuadamente a falta de textos escritos, salvo bajo el consabido argumento de la hipótesis de trabajo.

El análisis de los Sistemas Mundiales Antiguos, por el contrario, puede abrir una vía más empírica de aproximarnos a la posible presencia de relaciones de dependencia económica entre dos territorios más o menos distantes, por la presencia de relaciones de intercambio desiguales.

Para ello, el primer paso debería ser comenzar analizando los sistemas de comercio a escala comarcal, regional, nacional y supranacional que vayan aportando información positiva o negativa sobre los productos, cantidad y procedencia de las materias primas o artefactos objeto de tal tráfico, y que aporten nuevos elementos de discusión para ver si realmente será necesario o no modificar el actual paradigma peninsular rígidamente autoctonista, aislacionista y autosuficiente. La presencia de Sistemas Mundiales, si existieron, y concretamente, si la Península Ibérica estuvo integrada en alguno, surgirá como consecuencia de la información empírica acumulada sobre las redes de comercio y el carácter de las mismas, pero creemos que ha de dejarse una puerta abierta a la posibilidad de trabajar en el futuro, a modo de hipótesis de trabajo, sobre la presencia de un Sistema Mundial durante uno o varios periodos de nuestra Prehistoria Reciente. Si no asumimos la presencia de un único Sistema Mundial Antiguo, surge una visión más compleja, pero quizás más realista, que no depende simplemente de cuándo y cómo se produjo la integración dentro del Sistema Mundial único.

Resulta obvio que se trata de una estrategia arriesgada, pero aún así puede ser también prometedora. Un ejemplo claro es el periodo Bronce Final en las regiones meridionales de la Península Italiana, y particularmente Cerdeña, cuya visión en esta última década ha cambiado radicalmente (Vagnetti, 1993), frente a la noción estática previamente vigente. En cierta medida, la actual presunción de la necesidad de esperar hasta el periodo de las colonizaciones fenicia y griega en el litoral mediterráneo y atlántico de la Península Ibérica para la integración ibérica en las redes de comercio mediterráneas, donde las poblaciones indígenas representan el papel de 
receptores pasivos de los productos fabricados en regiones centrales como la costa siria-palestina, también implica asumir un enfoque conservador de partida.

Pero además. el estudio de los Sistemas Mundiales Antiguos aporta otras nociones implícitas, pero fundamentales. Es el análisis de los procesos históricos desde una perpectiva a largo plazo en el tiempo, pero también, a gran escala en el espacio. Puede resultar en ocasiones una minusvaloración de las variaciones locales o regionales, pero por el contrario, intenta siempre superar las actuales barreras provinciales, regionales y nacionales. Esa permanente creación de fronteras políticas de estudio donde en su momento no existieron, como entre Almería y Murcia, Tarragona y Zaragoza, Gerona y Rosellón, Pontevedra y Minho o Alentejo y Extremadura, por citar algunas. Pero también Córcega y Cerdeña, Malta y Sicilia o Epiro y Albania, por reseñar otras fuera de nuestras fronteras. Es en suma, preguntarnos si pueden llegar a existir crisis macroregionales, porque hay simultáneamente desarrollos regionales en unas áreas y crisis en otros territorios vecinos anteriormente pujantes, e incluso, si ciertas áreas pueden llegar a expandirse económicamente a expensas de otras dependientes comercial o políticamente.

\section{AGRADECIMIENTOS}

Queremos agradecer los comentarios al texto de R.J. Harrison, y de dos evaluadores del Comité de Redacción de T.P. que revisaron el primer y segundo manuscrito.

\section{BIBLIOGRAFÍA}

AMIN. S. (1974): «La acumulación a escala mundial». Siglo XXI. Madrid. $1^{\text {a }}$ ed. 1970.

BARAN. P. (1959): "La economía política del crecimiento". Fondo de Cultura Económica. México D.F. $1^{\text {a }}$ ed. 1957.

BouzeK, J. (1985): "The Aegean, Anatolia and Europe: Cultural Interrelations during the Second Millenium B.C.». Studies in Mediterranean Archaeology, 29. Paul Aströms Förlag, Jonsered.

BRAUDEL, F. (1994): «El Mediterráneo y el mundo mediterráneo en la época de Felipe II». Fondo de Cultura Económica. México. $1^{\mathrm{a}}$ ed. 1949.

BRUN, P. (1987): «Princes et princesses de la celtique. Le premier Age du Fer (850-450 av. J.C.)». Errance. Paris.

CANN, J.R. y RENFREW, A.C. (1964): "The characterization of obsidian and its application to the Mediterranean region". Proceedings of the Prehistoric Society, 30: 111 13

CINIIfFE. B.W. (1988): “Greeks, Romans and Barbarians. Sphere's of Interaction". B.T. Batsford. London.

(Hampion. T. (ed.) (1989): "Centre and Periphery". Comparative Studies in Archaeology. London.

CHIL.DE. V.G. (1954): "Los origenes de la civilización". Fondo de Cultura Económica. México D.F. 1 ${ }^{\text {a }}$ ed. 1936.

- (1978): "La prehistoria de la sociedad europea". Icaria. Barcelona. $1^{\text {a }}$ ed. 1958.

- (1986): "Nacimiento de las civilizaciones orientales". Editions 62. Barcelona. $1^{a}$ ed. 1934.

DAI.TON. G. (1976): “Teoría económica y sociedad primitiva". En M. Godelier (ed.): "Antropología y Economia". Anagrama. Barcelona: 179-207. $1^{\text {a }}$ ed. 1961.

EDENS. C. (1992): "Dynamics of Trade in the Ancient Mesopotamian “World System”». American Anthropologist, 94/1: 118-139.

EKHOLM. K. y FRIEDMAN. J. (1979): “Capital» imperialism and explotation in ancient world systems". En M.T. Larsen (ed.): "Power and Propaganda. A symposium on ancient empires". Mesopotamia. 7. Akademisk Forlag. Copenhagen: 41-58.

EMmanuel.. A. (1973): "El intercambio desigual". Siglo XXI. Madrid. $1^{\text {a }}$ ed. 1969.

FrANK. A.G. (1973): "Capitalismo y subdesarrollo en America Latina”. Siglo XXI. Buenos Aires. $1^{\text {a }}$ ed. 1969.

- (1979): “La acumulación mundial, 1492-1789". Siglo XXI. Madrid. $1^{\text {a }}$ ed. 1978.

- (1993): «Bronze Age World System Cycles». Current Anthropology, 34/4: 383-429.

FrankenSTEIN. S. y RowlandS, M.J. (1978): «The internal Estructure and regional context of Early Iron Age society in south- western Germany". University of London Institute of Archaeology Bulletin, 15: 74-112.

Gale. N.H. y Stos-Gale. Z.A. (1982): «Bronze Age Copper Sources in the Mediterranean: A New Approach». Science, 216: 11-19.

Gills. B.K. y FranK. A.G. (1990): «The cumulation of accumulation: theses and research agenda for 5000 years of world system history». Dialectical Anthropo$\log y, 15: 19-42$.

HARDING. A.F. (1984): "The Mycenaeans and Europe». Academic Press. London.

Herskovitz. M.J. (1954): "Antropología Económica". Fondo de Cultura Económica. México D.F. 1. ${ }^{a}$ ed. 1940.

Junghans, S.: SANGMEISTER. E. y SCHRÖDER, M. (1960): Metallanalysen kupferzeitlicher und frühbronzezeitlicher Bodenfunde aus Europa. Studien zu den Anfängen der Metallurgie, 1. Berlin.

KOHL, P.L. (1978): «The balance of trade in southwestern Asia in the mid-third millennium». Current Anthropology, 19: 463-492.

- (1987): "The ancient economy, transferable technologies and the Bronze Age world-system: a view from the northeastern frontier of the Ancient Near East». En M. Rowlands, M.T. Larsen y K. Kristiansen (eds.): «Centre and Periphery in the ancient world». New Directions in Archaeology. Cambridge University Press. Cambridge: 13-24. 
-. (1989): "The use and abuse of world systems theory: the case of the "pristine" west Asian state". En C.C. Lamberg-Karlovsky (ed.): "Archaeological Thought in America". Cambridge University Press. Cambridge. Mass.: 218-240.

LAMBER(i-KARLOVSKY. C.C. y TOSI, M. (1973): "Shahr-iSokhta and Tepe Yahya: Tracks on the earliest history of the Iranian Plateau». East and West, 23: 21-53.

Larsen, M.T. (ed.) (1979): "Power and Propaganda. A symposium on ancient empires". Mesopotamia, 7. Akademisk Forlag. Copenhagen.

LECLAIR. E.E. Jr. y SCHNEIDER. H.K. (1957): "Economic Anthropology». Molt. Rinehart. New York.

LENIN. V.I. (1960): "El Imperialismo, estadio supremo del capitalismo». Ediciones en Lenguas Extranjeras. Moscú.

LIVERANI, M. (1987): "The collapse of the Near Eastern regional system at the end of the Bronze Age: the case of Syria". En M. Rowlands, M.T. Larsen y K. Kristiansen (eds.): "Centre and Periphery in the ancient world". New Directions in Archaeology. Cambridge University Press. Cambridge: 13-24.

LUXEMBURG, R. (1967): «La acumulación del capital». Grijalbo. México D.F.

MARTín DE LA CRUZ, J.C. (1987): «¿Cerámicas micénicas en Andalucía?». Revista de Arqueología, 78: 62-64.

MARX. K. (1959): «El Capital». Fondo de Cultura Económica. México D.F.

NEWTON R.G. y RENFREW. A.C. (1970): «British faience beads reconsidered». Antiquity, 44, 175: 199-206.

PAILES, R. y WHITECOTTON, J. (1979): «The greatear southwest and mesoamerican "world" system: an exploratory model of frontier relationships». En W. Savage y S. Thompson (eds.): "The frontier: comparative studies». University of Oklahoma Press. Norman: 105-121.

Polanyi, K.; Arensberg, C.M. y Pearson, H.W. (eds.) (1976): "Comercio y Mercado en los Imperios Antiguos». Labor. Barcelona. $1^{\text {a }}$ ed. 1957.

RANDSBORG. K. (1991): "The First Millennium A.D. in Europe and the Mediterranean: An Archaeological Essay». Cambridge University Press. Cambridge.

- (1992): «Barbarians, classical antiquity and the rise of western Europe: an archaeological essay». Past and Present, 137: 8-24.

RENFREW, A.C. (1968): "Wessex without Mycenae». Annual of the British School of Archaeology at Athens, 63: $277-286$
- (1972): "The Emergence of Civilization: the Cyclades and the Aegean in the Third Millenium B.C.n. Methuen. London.

RENFREW. A.C. y PlaceY. J. (1968): "Aegean marble: a petrological study". Annual of the British School of Archaeology at Athens, 63: 45-66.

Rowl ANDS. M.: LARSEN, M.T. y KRISTIANSEN, K. (eds.) (1987): "Centre and Periphery in the ancient world". New Directions in Archaeology. Cambridge University Press. Cambridge: 13-24.

SCHNEIDER. J. (1977): «Was There a Pre-capitalist WorldSystem». Peasant Studies, 6: 20-29.

Sherratt, A. (1993)a: "Who are you Calling Peripheral? Dependence and Independence in European Prehistory». En C. Scarre y F. Healy (eds.): "Trade and exchange in prehistoric Europe». Oxbow Monographs. 33. Oxford: $245-255$.

- (1993)b: «What would a Bronze-Age World System look like?. Relations between temperate Europe and the Mediterranean in later prehistory". Journal of European Archaeology, 1/2: 1-57.

SHERRATT. A. y S. (1991): «From luxuries to commodities: The Nature of Mediterranean Bronze Age Trading Systems". En N.H. Gale (ed.): "Bronze Age trade in the Mediterranean". Studies in Mediterranean Archaeology, 90: 351-386. Jonsered.

ShERRATT, S. y A. (1993): "The growth of the Mediterranean economy in the early first millenium B.C.». World Archaeology, 24/3: 361-378.

Sweezy, P.M.; DobB, M.; TAKahashi. R.; Hilton, R. y HILL, C. (1977): «La transición del feudalismo al capitalismo». Crítica. Barcelona. $1^{\text {a }}$ ed. 1963.

Ucko, P. (ed.) (1995): "Theory in Archaeology». Routledge. London.

VAGNETTI, L. (1993): «Mycenaean pottery in Italy: fifty years of study». En C. y P. Zerner y J. Winder (eds.) "Wace and Blegen. Pottery as evidence for trade in the Aegean Bronze Age». J.C. Gieben. Amsterdam:143154.

WALLERSTEIN, I. (1979): «El moderno sistema mundial. I". Siglo XXI. Madrid-México D.F. $1^{\text {a }}$ ed. 1974.

- (1988): «El capitalismo histórico». Siglo XXI. Madrid. $1^{\text {a }}$ ed. 1983.

- (1991): "World system versus world-systems: A critique». Critique of Anthropology, 11: 